anales de psicología, 2013, vol. 29, n 3 (octubre), 810-815 http://dx.doi.org/10.6018/analesps.29.3.175701
(C) Copyright 2013: Servicio de Publicaciones de la Universidad de Murcia. Murcia (España) ISSN edición impresa: 0212-9728. ISSN edición web (http://revistas.um.es/analesps): 1695-2294

\title{
Factores de riesgo y protectores en el suicidio. Un estudio de casos y controles mediante la autopsia psicológica
}

\author{
José M. Antón-San-Martín ${ }^{1 *}$, Eduardo Sánchez-Guerrero², Lucía Pérez-Costilla 2,3, $\mathrm{M}^{2}$-Teresa Labajos-Manzanares², Yolanda \\ de-Diego-Otero ${ }^{4}$, Nicolás Benítez-Parejo ${ }^{5}$, Raquel Comino-Ballesteros ${ }^{4}$, Emilio Perea-Milla ${ }^{5}$, \\ Valentín Ramos-Medina ${ }^{6}$ y Amalia López-Calvo ${ }^{6}$

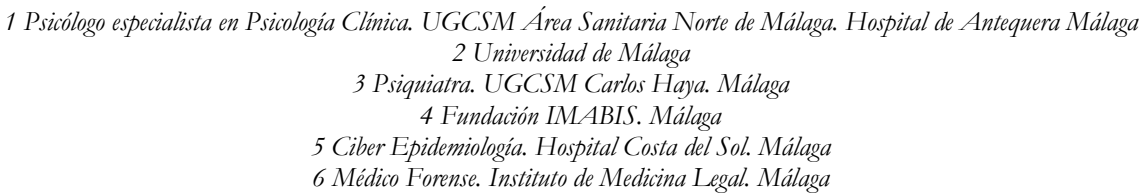

Resumen: Las tasas, los métodos y algunos de los factores de riesgo en el suicidio parecen depender de las diferencias geográficas y culturales, lo que implica la elaboración de estudios en zonas específicas de alto riesgo cara a la implementación de estrategias preventivas. Este estudio busca determinar los factores de riesgo y protectores en el suicidio en la Comarca de Antequera-Málaga (España). Se realiza en un estudio de caso y controles mediante la autopsia psicológica con 40 casos de suicidio y 80 controles poblacionales emparejados en género, edad y zona de residencia. El modelo multivariante hallado está compuesto por los siguientes factores: el presentar antecedentes familiares de suicidio y agregación de desordenes mentales en la familia, un diagnóstico de trastorno de la personalidad y conflictos familiares en el mes previo al suicidio. Estos factores permiten discriminar poblaciones de riesgo cara a la implementación de programas preventivos. Palabras clave: Suicidio; factores de riesgo; autopsia psicológica; estudio casos y controles.

\section{Introducción}

El suicidio es un importante problema de salud pública, encontrándose entre las primeras causas de muerte. La O.M.S. (2008) refiere que en el mundo se producen un millón de muertes por suicidio al año y cada suicidio afecta directamente a unas 6 personas. Señala así mismo, (O.M.S., 2003) la presencia de factores protectores y de riesgo de características universales dentro de la dinámica del suicidio. Los diversos acontecimientos o circunstancias estresantes como el desempleo, las pérdidas de personas queridas, las discusiones con la familia o los amigos, la ruptura de relaciones, los problemas legales, laborales y financieros pueden aumentara el riesgo de suicidio (Platt, 1984; Cavanagh, Owens y Johnstone, 1999a; Thacore y Varma, 2000; Schneider et al., 2011). El abuso de alcohol y drogas, los antecedentes de abusos físicos y/o sexuales en la infancia, el aislamiento social (Murphy y Welzel, 1990; Brown, 1999; Qin, Agerbo y Mortensen, 2003), los trastornos mentales (Harris y Barraclough, 1997; Cavanagh, Carson, Sharpe y Lawrie, 2003; ArsenaultLapierre, Kim y Turecki, 2004) y las enfermedades somáticas incapacitantes son considerados factores de riesgo (De Leo et al., 1999; Manoranjithan et al., 2010). Habría que añadir las tentativas de suicidio previas (Moscicki, 1997) y una historia familiar de suicidio y/o enfermedad mental (Runeson, 1998;

* Dirección para correspondencia [Correspondence address]: José Miguel Antón San Martín. Unidad de Salud Mental Comunitaria. Hospital de Antequera. Av. del Poeta Muñoz Rojas 29200 Antequera. Málaga (España).E-mail: jmasanmartin@telefonica.net
Title: Risk and protective factors in suicide. A case-control study using the psychological autopsy.

Abstract: Rates, methods and some of the risk factors of suicide seem to depend on geographical and cultural differences, which involve conducting research in specific high-risk areas facing the implementation of preventive strategies. This study seeks to determine the risk and protective factors of suicide in the region of Antequera-Málaga (Spain). Is performed on a casecontrol study using the psychological autopsy of 40 cases of suicide and 80 population controls matched on gender, age and area of residence. The multivariate model found is composed of the following factors: the antecedents of suicide and mental disorders aggregate in families, a diagnosis of personality disorder and family conflict in the month prior to suicide. These factors discriminate risk populations facing the implementation of preventive programs.

Key words: Suicide; risk factors; psychological autopsy; case-control study.

Runeson y Asberg, 2003; Kim et al., 2008). Los factores protectores encontrados son la participación en actividades religiosas y comunitarias, el tener niños pequeños y un seguimiento médico continuado (Kposowa, 2000; Qin y Mortensen, 2003).

La mayor dificultad para la comprensión de los suicidios es que no se puede entrevistar a los sujetos y por lo tanto no se puede comprobar directamente la razón del acto. Una solución encontrada ha sido el desarrollo del método denominado la autopsia psicológica. Se considera como el método por excelencia de recuperación de información postmorten (Isometsa, 2001; Cavanagh, Carson, Sharpe y Lawrie, 2003).

Nos planteamos estudiar el fenómeno suicida en la Comarca de Antequera -Málaga (España). Un área principalmente rural situada geográficamente en el extremo norte de la provincia de Málaga, lindando con las provincias de Córdoba y Sevilla, abarcando un total de 18 pueblos. Presenta una extensión de $2141.2 \mathrm{Km}^{2}$. Su población asciende a 103734 habitantes (I.N.E., 2001) y presenta una economía eminentemente agrícola y ganadera.

En un estudio previo sobre las tasas de suicidios en la zona (Antón, Gil y Navarro, 1992) se obtuvieron tasas brutas anuales en algunos pueblos de hasta 48.4 por 100000 habitantes. Se considera una de las zonas de Andalucía con las tasas más altas de suicidio (Consejería de Salud, 1998; Ocaña, Mayoral, Sánchez, Toro, Fernández y Méndez, 2008).

Ya la O.M.S (2003) señala la necesidad de controlar las tendencias epidemiológicas del suicidio en zonas geográficas determinadas con el objetivo de la realización de estudios 
que determinen que grupos poblacionales corren mayor riesgo cara a la implementación de programas preventivos que atajen o a lo sumo disminuyan dichas conductas suicidas.

Se realiza un estudio de casos y controles poblaciones mediante el método de la autopsia psicológica con el objetivo de averiguar qué factores, tanto de riesgo como protectores, presentan esta población.

\section{Método}

\section{Participantes}

Partiendo del listado de los suicidios producidos en la Comarca de Antequera emitido por el Instituto de Medicina Legal de Málaga entre el 1 de Enero del 2004 al 31 de diciembre del 2008 se recogieron 40 suicidios sucesivos. El grupo de controles $(n=80)$ fue seleccionado de forma aleatoria entre la población de la zona a partir del inicio del estudio. Los sujetos tanto en el grupo de casos como de controles poblaciones son mayores de 18 años. Por cada caso de suicidio se han obtenido dos controles (relación 1/2) emparejado por género, edad ( \pm 2 años) y zona geográfica.

\section{Procedimiento}

El contacto con familiares del suicidado se realizaba vía telefónica o a través del personal de la Zona Básica de Salud de procedencia (médico o personal de enfermería). Las entrevistas se realizaban en el domicilio, en dependencias del Centro de Salud o en el Hospital de Antequera, de acuerdo a la preferencia de los familiares.

Los controles fueron reclutados de forma aleatoria por parte del personal investigador y de los médicos de familia de los distintos centros de salud del Área Sanitaria. Se usó la base de datos del usuario (BDU) del Servicio Andaluz de Salud y los listados de consultas diarias de los mismos.

En las entrevistas, y una vez informado del estudio, se les solicitaba su colaboración y la firma del consentimiento informado. Posteriormente se pasaba a la realización de la autopsia psicológica y los instrumentos clínicos usados, para los familiares de los casos y los instrumentos clínicos solamente para los controles.

\section{Instrumentos clínicos}

\section{La autopsia psicológica}

Es un método cualitativo de recogida de información sobre la persona fallecida por suicido. Se realiza una entrevista con sus familiares y una revisión de su historia clínica. Se recogían las siguientes variables: Variables sociodemográficas: edad, sexo, estado civil, tipo de convivencia, nivel educativo, profesión, situación laboral, creencias religiosas y participación comunitaria. Características personales y familiares: método de suicidio, enfermedad somática crónica presente, tentativas de suicidio previas individual y familiar, antecedentes familiares de suicidio, presencia de enfermedad mental en familiares de primer orden (agregación familiar), presencia de niños pequeños y separación paterna temprana. Factores psicopatológicos: presencia de trastorno mental, diagnóstico y diagnóstico principal.

\section{Escala de Acontecimientos Vitales de Paul Ramsey (St. Paul Ramsey Life Experience Scale) (Rush, First y Blacker, 2000)}

Recoge un listado de experiencias, problemas y grado de impacto sobre la persona evaluada en el último mes. Analiza seis tipos de experiencias o acontecimientos: conyugales, interpersonales, laborales, de salud, vitales y otros. Cada suceso recibe una puntuación de gravedad que abarca desde el 1 (ninguno) a 7 (catastrófico).

Family Interview for Genetic Studies (FIGS) (Maxwell, 1992; Diaz de Villalvilla et al., 2008)

Es una entrevista semiestructurada enfocada a reunir información diagnóstica de un sujeto a través de sus familiares. Está diseñada para contribuir a una evaluación completa y con fines de investigación en los estudios de familiares en psiquiatría. Se realizan preguntas generales de cribado referentes a todos los familiares conocidos, y basándose en las respuestas se administra una hoja de perfil con una o más listas de síntomas, en este caso, para el sujeto suicida.

\section{Análisis de los datos}

Se usaron medidas de tendencia central y de dispersión para las variables cuantitativas, chi cuadrado y t-student como pruebas de contraste de hipótesis y distribución de frecuencias para las variables cualitativas. Para estimar la magnitud de las asociaciones se llevó a cabo un análisis univariado mediante modelos de regresión logística simple no condicionada para la variable caso/control con estimación de odds ratios y sus correspondientes intervalos de confianza al 95\%, estableciendo el nivel de significación en $p<.05$. Se han dicotomizado las variables para los análisis. En un segundo momento se realizó un modelo logístico multivariado (step forward) con aquellas variables que mostraron significación en el análisis univariado. Las variables se introdujeron en el análisis siguiendo la secuencia temporal de: variables sociodemográficas, familiares, personales/individuales y por último los sucesos vitales adversos sufridos en el último mes.

Los datos fueron procesados utilizando los programas informáticos estadísticos SPSS. Versión 15.0 para Windows.

\section{Resultados}

De los 40 casos, $27(67.5 \%)$ son hombres y $13(32.5 \%)$ son mujeres, con un ratio aproximado de $2 / 1$. La media de edad es de 56.2 años con una DT $=19.53$ (edad mínima 19-edad máxima 90) para el grupo de casos y de 55.91 años $(D T=$ 18.94) para el grupo control. 
El método más usado para suicidarse fue el ahorcamiento: $31(77.5 \%)$, seguido por el disparo: $4(10 \%)$.

Las variables sociodemográficas que resultaron ser factores de riesgo asociadas al suicidio son el vivir solo $(O R=$ 4.259; $\mathrm{IC}=1.069$ - 19.184) y el no trabajar $(\mathrm{OR}=4.58$; $\mathrm{IC}=$ 2.018 - 10.397). Un bajo nivel de estudios (sin estudios y es- tudios primarios incompletos) no está asociado al suicidio ( $p$ $=.116)$. Ni las creencias religiosas, ni el pertenecer a asociaciones comunitarias están asociados a factores protectores de suicidio. Las creencias junto con las prácticas religiosas aparece asociado como factor protector de suicidio $(O R=.286$; IC $=.0129-.63 ; p=.002)($ Tabla 1$)$.

$\underline{\text { Tabla 1. Modelo de regresión logístico univariado de los factores de riesgo y protectores de suicidio según las variables sociodemográficas. }}$

\begin{tabular}{|c|c|c|c|c|c|}
\hline & Suicido $N=40$ & Controles $N=80$ & OR & IC $(95 \%)$ & $p$ \\
\hline \multicolumn{6}{|l|}{ Factores de riesgo } \\
\hline Convivencia: Solo & $6(15 \%)$ & $3(3.8 \%)$ & 4.25 & $1.06-19.18$ & .04 \\
\hline Nivel bajo estudios* & $28(70 \%)$ & $44(55 \%)$ & 1.9 & $.85-4.27$ & .116 \\
\hline No trabaja & $28(70 \%)$ & $44(55 \%)$ & 4.58 & $2.01-10.39$ & .000 \\
\hline \multicolumn{6}{|l|}{ Factores protectores } \\
\hline Creencias religiosas & $38(95 \%)$ & $78(97.5 \%)$ & .48 & $.196-1.446$ & .216 \\
\hline Creencias y prácticas religiosas & $16(40 \%)$ & $56(70 \%)$ & .286 & $.129-.63$ & .002 \\
\hline Miembro asociación & $3(3.8 \%)$ & $3(3.8 \%)$ & 2.08 & $.401-10.81$ & .383 \\
\hline
\end{tabular}

*Agrupa las categorías de sin estudios y estudios primarios incompletos.

La presencia de una enfermedad crónica, las tentativas de suicidios previas, los antecedentes familiares de suicidio, la agregación familiar de enfermedad mental y una separación temprana materna y paterna aparecen como factores de riesgo asociados al suicidio. El tener niños menores de 6 años en el contexto familiar no se presenta como un factor protector.
El 87.5\% de los suicidios muestran agregación de enfermedad mental entre familiares de primer $\mathrm{o} / \mathrm{y}$ segundo grado frente al $13.8 \%$ en los controles. Aparecen en un $45 \%$ de los casos presentaron antecedentes familiares de suicidio de primer grado frente al 10\% del grupo de controles (ver Tabla 2).

Tabla 2. Modelo de regresión logístico univariado de los factores de riesgo y protectores de suicidio según las variables familiares y personales.

\begin{tabular}{|c|c|c|c|c|c|}
\hline & Suicidio $N=40$ & Controles $N=80$ & OR & $\mathrm{IC}(95 \%)$ & $p$ \\
\hline Antecedentes familiares de suicidio & $18(45 \%)$ & $8(10 \%)$ & 7.36 & $2.82-19.23$ & .000 \\
\hline Antecedentes familiares de tentativas de suicidio & $3(7.5 \%)$ & $0(0 \%)$ & & & \\
\hline Trastornos mentales en miembros familiares & $35(87.5 \%)$ & $11(13.8 \%)$ & 43.9 & $14.14-136.29$ & .000 \\
\hline Menores * & $1(2.5 \%)$ & $2(2.5 \%)$ & 1 & $.08-11.37$ & 1 \\
\hline Tentativas de suicidio previas & $13(32.5 \%)$ & $7(8.8 \%)$ & 5.02 & $1.81-13.91$ & .002 \\
\hline Enfermedad crónica & $14(35 \%)$ & $11(13.8 \%)$ & 3.37 & $1.36-8.38$ & .009 \\
\hline Separación temprana madre & $7(17.5 \%)$ & $3(3.8 \%)$ & 5.4 & $1.32-22.35$ & .019 \\
\hline Separación temprana padre & $8(20 \%)$ & $2(2.5 \%)$ & 9.75 & $1.96-48.44$ & .005 \\
\hline Separación temprana madre-padre & $5(12.5 \%)$ & $2(2.5 \%)$ & 5.57 & $1.03-30.11$ & .046 \\
\hline
\end{tabular}

*Tener hijos menores de 6 años

Del total de los casos, en $26(65 \%)$ aparece algún trastorno mental frente a $16(20 \%)$ del grupo de control. La presencia de desordenes mentales es un factor de riesgo en el suicidio consumado $(\mathrm{OR}=8.048 \mathrm{IC}=3.41-18.993 ; p<$ .000).Todos los grupos sindrómicos se pueden considerar factores de riesgo. El riesgo más elevado se presenta en los trastornos de personalidad $(O R=42.538$; $\mathrm{IC}=5.332$ 339.33; $p<.000)$ seguido de psicosis $(O R=16.758$; $\mathrm{IC}=$ $1.983-141.62 ; p<.010)$ (Tabla 3).

Tabla 3. Modelo de regresión logístico univariado de los factores de riesgo de suicidio según los diagnósticos sindrómicos*.

\begin{tabular}{|c|c|c|c|c|c|}
\hline & Suicido $N=40$ & Controles $N=80$ & OR & $\mathrm{IC}(95 \%)$ & $p$ \\
\hline Presencia de Trastorno Mental & $26(65 \%)$ & $16(20 \%)$ & 8.048 & $5.4 .41-18.93$ & .000 \\
\hline Trastornos Afectivos & $16(40 \%)$ & $11(13.8 \%)$ & 4.182 & $1.705-10.257$ & .002 \\
\hline Psicosis & $7(17.5 \%)$ & $1(1.3 \%)$ & 16.758 & $1.983-141.62$ & .010 \\
\hline Alcohol & $8(20 \%)$ & $4(5 \%)$ & 4.75 & $1.335-16.902$ & .016 \\
\hline Drogas & $3(7.5 \%)$ & $3(3.8 \%)$ & & & \\
\hline Trastornos de personalidad & $14(35 \%)$ & $1(1.3 \%)$ & 42.538 & $5.33-339.93$ & .000 \\
\hline
\end{tabular}

*Cada sujeto puede estar adscrito a más de una categoría.

Por grupos diagnósticos encontramos que son los trastornos del humor, con un $27.5 \%$, los más frecuentes, seguidos del grupo de las esquizofrenias con un $20 \%$, en el grupo de casos. Entre los sujetos del grupo control destacan los trastornos del humor con un 13.8\%. Señalar que el 35\% (n = 14) de los suicidas no presentaban diagnósticos psiquiátricos.

Cuando estudiamos los acontecimientos vitales estresantes en el mes previo al acto suicidio encontramos que este grupo muestra una $M=1.88$ sucesos $(D T=1.52)$ frente a 
una $M=0.4(D T=.805)$ del grupo de control $(\mathrm{t}=6.954, p$ $<.000)$. Por cantidad de sucesos, 16 suicidas $(40 \%)$ presentaron 3 o más sucesos frente al 3.8\% (n=3) del grupo control $\left(\mathrm{Ji}^{2}=36.44, p<.000\right)$. Señalar como en un $25 \%$ de los casos no aparecieron sucesos adversos previos al suicidio.
En la Tabla 4 se re presentan los sucesos vitales adversos que se pueden asociar como factores de riesgo en el suicidio. Por magnitud destacan los conflictos familiares $(O R=$ 15.643; IC $=5.75-42.55)$ y las dificultades económicas $(O R$ $=13 ; \mathrm{IC}=2.69-62.831)$.

Tabla 4. Modelo de regresión logístico univariado de los factores de riesgo de suicidio según los principales sucesos adversos*.

\begin{tabular}{lcccc}
\hline & Casos $N=40$ & Controles $N=80$ & OR & \multicolumn{1}{c}{ IC $(95 \%)$} \\
\hline Conflicto de pareja & $14(35 \%)$ & $8(10 \%)$ & 4.846 & $1.823-12.881$ \\
Conflictos familiares & $24(60 \%)$ & $7(8.75 \%)$ & 15.643 & $5.75-42.55$ \\
Conflictos laborales & $7(17.5 \%)$ & $2(2.5 \%)$ & 8.273 & $1.632-41.942$ \\
Dificultades financieras & $10(25 \%)$ & $2(2.5 \%)$ & 13 & $2.69-62.831$ \\
Enfermedad familiares & $9(22.5 \%)$ & $2(2.5)$ & 11.323 & .000 \\
\hline
\end{tabular}

*Cada sujeto puede puntuar en más de una categoría.

Después del análisis de regresión logística multivariado realizando teniendo en cuenta el sexo y la edad, del total de variables analizadas solamente cuatro de ellas han entrado en el modelo: el tener antecedentes familiares de suicidio, los trastornos mentales en la familia, un diagnóstico de trastor- nos de personalidad y los conflictos familiares en el mes previo al suicidio. No se han hallado interacciones significativas en el resto de las variables independientes tenidas en cuenta (Tabla 5).

Tabla 5. Modelo de regresión logístico multivariado de los factores de riesgo y protectores de suicidio.

\begin{tabular}{|c|c|c|c|c|c|}
\hline & Casos $N=40$ & Controles $N=80$ & OR & IC $(95 \%)$ & $p$ \\
\hline Antecedentes familiares de suicidio & $18(45 \%)$ & $8(10 \%)$ & 9.947 & $21.707-57.97$ & .011 \\
\hline Trastornos mentales en miembros familiares & $35(87.5 \%)$ & $11(13.8 \%)$ & 13.33 & $4.315-41.183$ & .000 \\
\hline Trastorno de personalidad & $14(35 \%)$ & $1(1.3)$ & 38.052 & $1.190-1216.93$ & .04 \\
\hline Conflictos familiares & $24(60 \%)$ & $7(8.75 \%)$ & 7.087 & $1.354-37.096$ & .02 \\
\hline
\end{tabular}

\section{Discusión y Conclusiones}

La media de edad del grupo de suicidas fue de 56.2 años, similar a la encontrada en estudios descriptivos en distintos lugares de España, no así en estudios internacionales usando la misma metodología (Cavanagh, Owens y Johnstone, 1999; Phillips et al., 2002; Zhang, Conwell, Zhou y Jiang, 2004; Palacio et al., 2007). Lo que indica que nuestra población suicida está formada principalmente por personas de mayor edad como característica diferenciadora con respecto a otros países.

Las creencias religiosas asociado a las prácticas que estas conllevan aparece como un elemento protector con respecto al riesgo suicida. Este dato es hallado en el estudio de Neeleman y Lewis (1999). Este factor de protección puede estar relacionado con las mismas creencias religiosas y la valoración que del acto suicida como tal tienen y por otra parte, las prácticas religiosas, aparte del ritual que supone, presenta un alto componente de integración social y participación en actividades comunitarias. Es cierto, que en nuestra población el participar en actividades comunitarias no aparece como elemento protector, al contrario que en otros trabajos como los de Rubenowitz et al. (2001) y de Duberstein, Conwell, Conner, Eberly, Evinger y Caine (2004) donde si aparece.

Con respecto al resto de las variables definidas como sociodemográficas solamente aparecen asociadas al suicidio el vivir solo y el no trabajar. Éste último resultado está condicionado por la edad de la población, con lo cual solamente podemos considerar la variable convivir solo como característica de riesgo. De Leo et al. (1999) encontró este mismo resultado.

El tener hijo pequeños, sobre todo menores de 6 años y dependientes de los padres, ha sido otra variable asociada a protección frente al riesgo suicida (Qin y Mortensen, 2003; Qin et al., 2003) que no ha aparecido en este estudio. Como se ha señalado anteriormente, nuestra población de personas suicidadas presenta una edad muy superior al resto de estudios. Más del $50 \%$ de los suicidas superan los 56 años, con lo cual desde un punto de vista evolutivo se encuentran lejos de tener hijos pequeños.

Las tentativas previas de suicidio es un factor asociado al suicidio, encontrado en otras investigaciones independientemente del país de la realización del mismo y de la muestra utilizada (Phillips et al., 2002; Chiu et al., 2004; Gurujag, Isaac, Subbakrishna y Ranjani, 2004; Chen et al, 2006; Zonda, 2006; Li, Phillips, Zhang, Xu y Yang, 2008). También aparece en nuestro estudio. Al igual que en el estudio de $\mathrm{Pa}$ lacio et al, (2007) la historia familiar de tentativas de suicidio no se asocia a riesgo de suicidio ya que se pueden considerar como dos fenómenos diferentes (Moscicki, 1997).

La historia de suicidios en la familia es uno de los indicadores más intensos de riesgo. Es un factor de riesgo presente, tanto con poblaciones con adolescentes y adultos (Cheng et al., 2000; Rubenowitz et al., 2001; Qin et al., 2002; Qin et al, 2003; Runeson y Asberg, 2003; Palacio et al., 2007), como con muestras con distintas patologías psiquiátricas (McGirr et al., 2006). Los estudios sobre adopción, gemelos y de fa- 
milia apoyan la opinión que la etiología de la transmisión familiar de comportamiento suicida es al menos en parte, genética, y puede ser mediada por la transmisión de fenotipos intermedios, como la agresión impulsiva (Brent y Mehlem, 2008). Además, puede haber causas ambientales para la transmisión familiar, incluyendo la imitación, y la transmisión intergeneracional del manejo y resolución de la adversidad en familia, así como un estilo particular de manejar los conflictos y los problemas, aprendido en la primera infancia.

Con una historia de psicopatología en familiares de primer orden ocurre algo similar que con la presencia de historia de suicidios a nivel familiar. Está continuamente presente en los estudios que valoran esta variable (Vjayakumar y Rajkumar, 1999; Qin et al, 2002; Qin et al., 2003). Junto con la anterior, son los factores asociados con más intensidad al riesgo de suicidio.

En este estudio, el porcentaje de trastorno mental en el grupo de casos fue del $65 \%$, porcentaje inferior al encontrado en otros estudios aunque similar al hallado por Phillips et al (2002) en China y por Owens et al. (2003) en Inglaterra. La variación en los mismos puede ser fruto de los instrumentos de medida y de diagnóstico, y de las variaciones socioculturales propias de cada país. Arsenault-Lapierre, Kim y Turecki (2004) en su meta- análisis de 3275 suicidios concluyen que las diferencias geográficas estaban presentes en las proporciones de trastornos mentales entre suicidios por una parte, y por otra como en Europa y China el principal diagnóstico entre suicidas son los trastornos afectivos, mientras que en Norteamérica y Australia es el de abuso de sustancias. Todos los desordenes emocionales asociados al suicidio están presentes en este estudio, aunque la intensidad varia de unos a otros. Son las psicosis junto con los trastornos de personalidad los que mayor magnitud de riesgo presentan.

Salvo el consumo de drogas, todos los desordenes emocionales asociados al suicidio están presentes en este estudio, aunque la intensidad del riesgo varía de unos a otros.

La presencia de acontecimientos vitales adversos es un importante factor de riesgo para el suicidio (Cavanagh, Owens y Johnstone, 1999a; Rubenowitz et al., 2001; Phillips et al., 2002; Owens, Booth, Briscoe, Lawrence y Lloyd, 2003; Gurujag et al., 2004; Zhang et al., 2004; Chen et al., 2006;

\section{Referencias}

Antón, J., Gil, E. y Navarro, F. (1992). El suicidio y su evolución en el Distrito Sanitario de Antequera. 1986-1991. Maristán. Revista de la Asociación Andaluza de Neuropsiquiatría, 3, 7-15.

Arsenault-Lapierre, G., Kim, C. y Turecki, G. (2004). Psychiatric diagnosis in 3275 suicides: A meta-analysis. BMC Psychiatry, 4, 37-47.

Ayuso, J., Baca, E., Bobes, J., Giner, J., Giner, L., Pérez, V., Sáiz, P., Saiz, J. y Grupo RECOMS. (2012). Recomendaciones preventivas y manejo del comportamiento suicida en España. Revista de Psiquiatría y Salud Mental. 5(1) 8-23.

Brent, D. y Mehlem, N. (2008). Familial transmission of suicidal behavior. Psychiatric Clinics of North America, 31,157-77.

Brown, J. et al. (1999). Childhood abuse and neglect specificity of effects on adolescent and Young adult depression and suicidality. Journal of the American Academy of Child and Adolescent Psychiatry, 38, 1490-1496.
Zonda, 2006; Palacio et al., 2007; Overholser, 2012). Las dificultades financieras, los conflictos familiares y de pareja, los problemas laborales y el manejo de la enfermedad de un familiar son los principales acontecimientos adversos encontrados.

La presencia de más de un suceso o acontecimiento de vida adverso y su posible efecto acumulativo es un dato importante que define a nuestra población suicida, dato no hallado en las revisiones realizadas sobre los factores de riesgo en el suicidio y que sería necesario comenzar a considerar dentro del fenómeno suicida.

Cuando se evalúa el efecto combinado de todas las variables aparece un modelo explicativo del suicidio en nuestra población. La presencia de trastornos mentales en familiares de primer orden, con antecedentes familiares de suicidio, un diagnóstico de trastorno de personalidad, y conflictos familiares en el mes previo son factores que independientemente y en asociación constituyen un modelo inicial multifactorial de riesgo de suicidio. Modelo de factores marcado por el tipo de análisis realizado. El seguir una secuencia concreta de introducción de las variables viene definido por el criterio evolutivo/temporal de influencia de los factores en el curso vital del sujeto que ha consumado el suicidio. Por ejemplo, la influencia religiosa se considera como una variable contextual que ya está presente socialmente previa al sujeto mientras que los sucesos adversos son las últimas variables que inciden en el sujeto previo al suicidio.

No obstante y dado el reducido número de la muestra, es necesario continuar el proceso de recogida no solamente para la búsqueda de modelos predictivos del mismo sino para la estructuración de programas preventivos focalizados en la detección y abordaje de personas que posean dichos factores y por lo tanto consideradas como población de riesgo (Mann, Apter y Bertolonte, 2005; Ayuso et al., 2012).

Nota.- Los datos usados en este trabajo son parte de una investigación sobre el suicidio financiada por la Consejería de Salud (PI0154/2007; 143/05) y la Consejería de Innovación, Ciencia y Empresa (PICTS546). Junta de Andalucía y el Instituto de Salud Carlos III. Fondo de Investigaciones Sanitarias PI061339.

Cavanagh, J., Carson, A., Sharpe, M. y Lawrie, J. (2003). Psychological autopsy studies of suicide: A systematic review. Psychological Medicine, 33(3), 395-405.

Cavanagh, J., Owens, D. y Johnstone, E. (1999a). Life events in suicide and undetermined death in south-east Scotland: A case-control study using the method of psychological autopsy. Social Psychiatry and Psychiatric Epidemiology, 34, 645-650.

Cavanagh, J., Owens, D. y Johnstone, E. (1999). Suicide and undetermined death in south east Scotland. A case-control study using the psychological autopsy method. Psychological Medicine, 29, 1141-1149.

Chen, E., Chan, W., Wong, P., Chan, S., Law, Y., Beh, P., Chan, K., Cheng, J., Liu, K. y Yip, P. (2006). Suicide in Hong Kong: A case-control psychological autopsy study. Psychological Medicine, 36(6), 815-25. 
Chiu, H., Yip, P., Chi, I., Chan, S., Tsoh, J., Kwan, C.,... Caine, E. (2004). Elderly suicide in Hong Kong: A case-controlled psychological autopsy study. Acta Psychiatrica Scandinavica, 109(4), 299-305.

C.I.E. 10. Clasificación de los Trastornos Mentales y del Comportamiento. (2000). Madrid: Editorial Médica Panamericana.

Consejería de Salud. Junta de Andalucía (1998). Estadísticas Vitales: Distribución Espacial y Tendencia de la Mortalidad por Cáncer y otras Causas. Andalucía 1976-1996. Sevilla.

De Leo, D. et al. (1999). Physical illness and parasuicide: evidence from the European Parasuicide Study Interview (EPSIS/WHO-EURO). International Journal of Psychiatry in Medicine, 29, 149-163.

Díaz de Villalvilla, T., Mendoza, R., Martín, M., del Castillo, N., Bravo, T., Domínguez, M., y Vega, M. (2008). Versión en español de la entrevista familiar para estudios genéticos (FIGS). Actas Esp. Psiquiatr. 36(1,) 20 24.

Duberstein, P., Conwell, Y., Conner, K., Eberly, S., Evinger, J. y Caine, E. (2004). Poor social integration and suicide: fact or artifact? A casecontrol study. Psychological Medicine, 34(7), 1331-1334.

Gururaj, G., Isaac, M., Subbakrishna, D. y Ranjani, R. (2004). Risk factors for completed suicides: A case-control study from Bangalore, India. Injury Control and Safety Promotion, 11(3), 183-91.

Harris, E. y Barraclough, B. (1997). Suicide as on outcome for mental disorders. British Journal of Psychiatry, 170, 447-452.

Instituto Nacional de Estadística. www.ine.es/inebmenu/mnu_cifraspob.html.

Isometsa, E. (2001). Psychological autopsy studies. A review. Eur. Psychiatry, 16, 379-385.

Kim, C., Seguin, M., Thernien, N., Riopel, G., Chowky, N., Lesage, A. y Turecki, G. (2005). Familial aggregation of suicidal behavior: A family study of male suicide completers from the general population. American Journal of Psychiatr, 162, 1017-1019.

Kposowa, A. (2000). Marital status and suicide in the National Longitudinal Mortality Study. Journal of Epidemiology and Community Health, 54, 245-61.

Li, X., Phillips, M., Zhang, Y., Xu, D. y Yang, G. (2008). Risk factors for suicide in China's youth: a case-control study. Psychological Medicine, 38(3), 397-406.

Mann, J., Apter, A., Bertolonte, J., et al. (2005). Suicide prevention strategies. A systematic review. JAMA, 294(16), 2064-2074.

Manoranjithan, S., Rajkumar, A., Thangaduran, P., Prasad, J., Jayakayan, R. y Jacob K. (2010). Risk factors for suicide in rural south India. The British Journal of Psychiatry, 196, 26-30.

Maxwell, E. (1992). The Family Interview for genetic studies manual. Washington DC. National Institute of Mental Health, Intramural Research Program, Clinical Neurogenetics Branch.

McGirr, A., Tousignant, M., Routhier, D., Pouliot, L., Chawky, N., Margolese, H. y Turecki, G. (2006). Risk factors for completed suicide in schizophrenia and other chronic psychotic disorders: A case-control study. Schizophrenia Research, 84(1), 132-143.

Moscicki, E. (1997). Identification of suicide risk factors using epidemiologic studies. Psychiatric Clinics of North America, 20, 499-517.

Murphy, G. y Welzel, R. (1990). The life-time risk of suicide in alcoholism. Archives of General Psychiatry, 47, 383-392.

Neeleman, J. y Lewis, G. (1999). Suicide, religion, and socioeconomic conditions. An ecological study in 26 countries, 1990. Journal of Epidemiology and Community Health, 53, 204-210.
Ocaña, R., Mayoral, J., Sánchez, C., Toro, S., Fernández, A. y Méndez, C. (2008). Atlas interactivo de mortalidad en Andalucía (AIMA). Revista Española de Salud Pública, 82 (4), 379-394.

Organización Panamericana de la Salud, Oficina Regional para las Américas de la Organización Mundial de la Salud (2003). Informe Mundial sobre la Violencia y la Salud. Washington DC.

Organización Mundial de la Salud (2008). Preventing suicide a resource for media professionals department of mental health and substance abuse world bealth. Genove.

Overholser J. (2012).Understanding suicide risk: identification of high-risk groups during high-risk times. Journal of Clinical Psychology. 68(3), 349-61.

Owens, C., Booth, N., Briscoe, M., Lawrence, C. y Lloyd, K. (2003). Suicide outside the care of mental health services: A case-controlled psychological autopsy study. Crisis, 24(3), 113-21.

Palacio, C., García, J., Diago, J., Zapata, C., López, G., Ortiz, J. y López, M. (2007). Identification of suicide risk factors in Medellin, Colombia: A case-control study of psychological autopsy in a developing country. Archives of Suicide Research, 11, 297-308.

Platt, S. (1984). Unemployment and suicidal behavior: A review of the literature. Social Science and Medicine, 19, 93-115.

Phillips, M., Yang, G., Zhang, Y., Wang, L., Ji, H. y Zhou, M. (2002). Risk Factors for Suicide in China: A national case-control psychological autopsy study. The Lancet, 360(30), 1728-1736.

Qin, P., Agerbo, E. y Mortensen, P. (2003). Suicide risk in relation to socioeconomic, demographic and familial factors: A National Register-Bases Study of all suicides in Denmark. 1981-1997. American Journal of Psychiatry, 160(4), 765-772.

Qin, P. y Mortensen, B. (2003). The impact of parental status on the risk of completed suicide. Archives of General Psychiatry, 60, 797-802.

Rubenowitz, E., Waern, M., Wilhelmson, K., et al. (2001). Life events and psychosocial factors in elderly suicides-a case-control study. Psychological Medicine, 31, 1193-1202.

Runeson, B. (1998). History of suicidal behavior in the families of Young suicides. Acta Psychiatrica Scandinavica, 98, 497-501.

Runeson, B. y Asberg, M. (2003). Family history of suicide among suicide victims. American Journal of Psychiatry, 160, 1525-1526.

Rush, A., First, M. y Blacker, D. (2000). Handbook of Psychiatric Measures. Washington DC: American Psychiatric Publishing Inc.

Schneider, B., Grebner, K., Schnabel, A., Hampel, H., Georgi, R. y Seidler, A. (2011). Impact of employment status and work-related factors on risk of completed suicide. A case-control psychological autopsy study. Psychiatry Research. 190, 265-270.

Thacore, V. y Varma, S. (2000). A study of suicides in Ballarat, Victoria, Australia. Crisis, 21, 26-30.

Vijayakumar, L. y Rajkumar, S. (1999). Are risk factors for suicide universal? A case-control study in India. Acta Psychiatrica Scandinavica, 99, 407-411.

Zhang, J., Conwell, Y., Zhou, L. y Jiang, C. (2004). Culture, risk factors and suicide in rural China: A psychological autopsy case-control study. Acta Psychiatrica Scandinavica, 110(6), 430-37.

Zonda, T. (2006). One-hundred cases of suicide in Budapest. A case-control psychological autopsy study. Crisis, 27(3), 125-129.

(Artículo recibido: 25-11-2010; revisado: 26-12-2012; aceptado: 07-01-2013) 